\title{
Palhaços de hospital como estratégia de amenização da experiência de hospitalização infantil
}

\author{
Susana Caires - Universidade do Minho, Braga, Portugal \\ Carla Hiolanda Esteves - Susana Correia \\ Isabel Almeida - Universidade do Minho, Braga, Portugal
}

\begin{abstract}
Resumo
A hospitalização pediátrica pode representar um acontecimento marcante para a criança e sua família, dado o desenraizamento de seus contextos e rotinas, e a imersão num ambiente estranho e ameaçador. Buscando minimizar o impacto dessa experiência, várias abordagens lúdicas têm surgido em pediatria, nomeadamente a intervenção de palhaços. Assumindo que, mesmo hospitalizada, existe, na criança, uma essência que deseja brincar, a intervenção do palhaço tem como propósito o seu resgate. Neste artigo, apresentam-se as expetativas de profissionais pediátricos $(n=34)$ a respeito das (des)vantagens da presença dos palhaços junto dessas crianças/adolescentes, antes mesmo da sua intervenção. Os dados revelaram uma ampla abertura à presença desses artistas, apontando-os como potenciais amenizadores do impacto emocional da internação e dos tratamentos, e seu contributo para a humanização dos cuidados e desmistificação dos profissionais de saúde. Como desvantagens, referiram o medo do palhaço ou a percepção, entre adolescentes, da sua presença como uma infantilidade.
\end{abstract}

Palavras-chave: Pediatria; Palhaços de hospital; Ludicidade.

\section{Hospital clowns as a strategy for the mitigation of childhood hospitalization experience}

\begin{abstract}
Pediatric hospitalization may represent a significant event in the life of children and their families due to the uprooting of their contexts and routines, and the immersion in a strange and threatening environment. Seeking to minimize the impact of this experience, various ludic approaches emerged in pediatrics, namely the hospital clowns. Assuming that, even in the hospital, the child carries an essence that wants to play, the clowns' mission is to rescue it. This manuscript presents the staff's expectations regarding the (dis)advantages of clowning in their unit $(n=34)$, a month before starting intervention. Results reveal an expressive opening regarding the clowns' presence, pointing them as potential buffers of the emotional impact of hospitalization and treatments on children, and contributing to the humanization of care and demystification of health staff. Regarding setbacks, participants mentioned the children's fear of clowns and the perception - by adolescents - of their presence as childish. Keywords: Pediatrics; Hospital clowns; Play.
\end{abstract}

Payasos del hospital como estrategia de mitigación de la experiencia de hospitalización infantil

\begin{abstract}
Resumen
La hospitalización pediátrica puede representar un evento clave para los niños y sus familias, dado el desarraigo de sus contextos y rutinas, y la inmersión en un ambiente extraño y amenazante. Intentando minimizar el impacto de esta experiencia, diversos enfoques lúdicos han surgido en pediatría, incluyendo los payasos. Estas suponen que, aún hospitalizado, existe una esencia en el niño que quiere jugar. La misión del payaso es rescatarla. En este artículo se presentan las expectativas de 34 profesionales pediátricos en relación con las (des)ventajas de la presencia de payasos, un mes antes de iniciar su intervención. Los datos revelaron su amplia apertura a la presencia de payasos, señalándolos como potencialmente disminuyendo el impacto emocional de la hospitalización, del tratamiento, contribuyendo además a la humanización de los cuidados y desmitificación de los profesionales. Como desventajas, han mencionado el temor del payaso o la percepción, entre adolescentes, de su presencia como infantil. Palabras-clave: Pediatría; Payasos de Hospital; Jugar.
\end{abstract}

\section{Introdução}

A hospitalização pediátrica tem vindo a ser alvo de pesquisa nas últimas décadas, havendo evidências que a descrevem como um evento potencialmente marcante para a criança/adolescente e sua família (Barros, 1998; Parcianello \& Felin, 2008; Redondeiro, 2003). Vários estudos têm procurado identificar os principais fatores de estresse associados a esta experiência, a intensidade com que são experienciados, bem como o seu impacto no bem-estar físico e psicológico da criança/ adolescente, na qualidade do seu internamento, no processo de recuperação, e/ou seus efeitos desenvolvimentais (Bess d'Alcantara, 2008; Brewer, Gleditsch, Syblik, Tietjens, \& Vacik, 2006; Broering \& Crepaldi, 2011; Martins \& Paduan, 2010; Masetti, 1998, 2011; Parcianello \& Felin, 2008).

Dentre os principais fatores de estresse identificados, surgem i) o afastamento e separação da criança de seus principais contextos de vida (família, escola, grupo de amigos); ii) alterações significativas em suas rotinas; e iii) a percepção de ameaça perante o desconhecido, 
a dor e o desconforto associados à sua situação clínica, tratamentos e exames (Bess d'Alcantara, 2008; Linge, 2011; Martins \& Paduan, 2010; Mazur, Batista, Andreatta, Ribas, \& Campos, 2005; Parcianello \& Felin, 2008; Vieira \& Lima, 2002). Quanto às reações a esses estressores, o medo, a tristeza, angústia, insegurança, desconforto ou dor são frequentemente reportados (Mazur e cols., 2005; Vieira \& Lima, 2002).

Segundo Kumamoto e cols. (2004), as privações afetivas, cognitivas e lúdicas experienciadas pela criança acarretam, frequentemente, significativos níveis de sofrimento físico e psicológico que podem "precipitar ou agravar desequilíbrios psico-afetivos com repercussões importantes sobre a sua saúde já fragilizada" (Kumamoto e cols., 2004, s/ p). Alterações de sono, apetite, comportamento, humor ou no seu desempenho cognitivo e social são também habitualmente registadas, podendo representar obstáculos ao tratamento, à qualidade da recuperação (Broering \& Crepaldi, 2011; Fernandes \& Arriaga, 2010; Garcia, Silva, Vaz, Filocomo, \& Filipini, 2009; Schmitz, Piccoli, \& Vieria, 2003) e, entre outros, à duração da internação (Barros, 1998, p. 14). Em casos mais extremos, Parcianello e Felin (2008) descrevem tais experiências como "traumáticas" (p. 147).

Entre algumas abordagens que buscam minimizar o impacto e os efeitos da hospitalização na criança, evidenciamos não apenas as de foro médico e farmacológico, mas, também, as de âmbito psicológico e lúdico, pelo crescente reconhecimento dos benefícios dessas últimas para a criança e seus cuidadores (por exemplo: menor estresse, maior sentido de controle, estratégias de enfrentamento mais adequadas) e, consequentemente, a sua mais frequente recomendação entre as intervenções alternativas a serem promovidas em pediatria (Barros, 1998; Mitre \& Gomes, 2004; Motta \& Enumo, 2004). A criação de brinquedotecas em contexto hospitalar (Oliveira, Gabarra, Marcon, Silva \& Macchiaverni, 2009), de programas de contadores de histórias (Mussa \& Malerbi, 2008), música (Fernandes, 2008), magia (Hart \& Walton, 2010), ou palhaços de hospital (Caires e cols., 2010; Masetti, 2011) surgem entre algumas dessas recomendações.

Quanto às intervenções lúdicas em contexto hospitalar, estas são, atualmente, amplamente reconhecidas. De acordo com Melo (2007), brincar é um meio de expressão e integração da criança no ambiente circundante. É através da brincadeira que ela explora e conhece o mundo; aprende a lidar com suas emoções e sentimentos, e com os das outras pessoas. Brincar ajuda, também, a estabelecer relações entre o imaginário e a realidade, e constrói uma ligação entre seu próprio ser e o mundo de significados e objetos (Hockenberry, 2006; citado por Tavares, 2008). Pelas razões anteriormente referidas, vários autores assumem o brincar como a atividade mais importante para a criança, por ser essencial ao seu desenvolvimento psicomotor, emocional, mental e social (Masetti, 2003; Mitre \& Gomes, 2004; Tavares, 2008). Aplicando diretamente essas ideias ao contexto pediátrico, Mitre e Gomes (2003) afirmam que o brincar dá a oportunidade de disfarçar o dia a dia do hospital, produzindo uma realidade única que, alternando entre o imaginário e o mundo real, permite à criança transpor a barreira da doença, e os limites do tempo e do espaço.

Enfatizando o potencial desenvolvimental e terapêutico do lúdico em contexto hospitalar, Fridman (1996; citado por Motta \& Enumo, 2004) assume-o como uma experiência reestruturante que supera o sofrimento de um internamento; que aumenta a probabilidade de ultrapassar os potenciais traumas gerados pela internação, favorecendo, assim, o restabelecimento físico e emocional da criança que viveu essa experiência. No olhar de Fridman (1996, citado por Motta \& Enumo, 2004), brincar torna o ambiente hospitalar menos traumatizante e mais alegre, proporcionando momentos de higiene mental e, entre outros, a manutenção de uma relação estável entre a criança, sua família e a equipe de saúde. A humanização do atendimento, a estimulação do desenvolvimento psicossocial da criança e a prevenção da saúde mental dos diferentes intervenientes fazem também parte das vantagens apontadas à inserção do lúdico em contexto pediátrico (Lima, Azevedo, Nascimento, \& Rocha, 2009; Martins \& Paduan, 2010; Motta \& Enumo, 2004; Oliveira e cols., 2009; Redondeiro, 2003).

\section{Lúdico, humor e palhaços de hospital}

Conscientes das potencialidades do brincar ao nível do bem-estar, desenvolvimento e qualidade de vida da criança hospitalizada, alguns grupos de palhaços têm vindo a desenvolver uma intervenção específica nesses contextos. Klein (1993; citado por Wuo, 1999), tentando identificar o que poderá contribuir para os benefícios observados na intervenção dos Palhaços de Hospital $(\mathrm{PH})$, refere o humor e o brincar como nucleares. Em seu estudo, Klein assume que o intenso prazer vivido pelas crianças em suas brincadeiras com os $\mathrm{PH}$ resulta não apenas do prazer de brincar, mas, também, do fato de, ao brincar, encontrarem uma forma de dominar sua angústia. Segundo Gryski (2003), na interação entre a 
criança e PH, estes "work together to create a safe play place within the larger unfamiliar hospital environment" (p. 98). De entre os benefícios encontrados pela pesquisa nessa área destacam-se: as alterações (positivas) em áreas como apetite, sono, adesão da criança aos procedimentos e exames médicos, tratamentos e/ou interação com os profissionais de saúde, bem como na redução de seus níveis de ansiedade (por exemplo: Araújo \& Guimarães, 2009; Azevedo, Santos, Justino, Miranda, \& Simpson, 2007; Duffin, 2009; Linge, 2012).

Por exemplo, Golan e cols. (2009) e Fernandes e Arriaga (2010) verificaram que a presença dos $\mathrm{PH}$ no período pré-operatório diminui os níveis de ansiedade da criança e de seus pais, como também no pós-operatório (menor ansiedade, afetos mais positivos). Adicionalmente, salientam-se os efeitos positivos da intervenção dos $\mathrm{PH}$ na comunicação verbal e não verbal da criança; na atitude face a si própria, à doença e à hospitalização; o aumento da expressão de emoções como o riso, a alegria e o humor; ou o aumento de sua percepção de controle, autoconfiança e vitalidade, bem como uma maior abstração da doença e dos problemas vividos em volta da mesma (Garcia e cols., 2009; Gryski, 2003; Kingsnorth, Blain, \& McKeever, 2010; Linge, 2011, 2012; Masetti, 1998, 2011; Motta \& Enumo, 2004). Linge (2011), por seu lado, afirma que o palhaço "infecta" a criança com afetos positivos, assim como todos aqueles que estão envolvidos na atividade.

Vale salientar, porém, referências feitas aos medos da criança em relação ao palhaço, especialmente nos primeiros contatos (Gryski, 2003; Linge, 2012). A presença/ausência dos pais quando da visita dos $\mathrm{PH}$, a idade e/ou o gênero da criança, as características do hospital ou o tempo de internação (mais extenso no caso de crianças com doenças crônicas, com internamentos e/ou visitas repetidas ao hospital) são apontados como potenciais diferenciadores dessas reações (Meisel e cols., 2009). Quanto ao fator “idade", por exemplo, Linge (2012) verificou em seus estudos que, entre a grande parte dos adolescentes entre os 14 e os 18 anos, apesar de assumirem a presença dos $\mathrm{PH}$ como contribuindo para a quebra das rotinas hospitalares e como podendo "lighten up the mood and give some relief from the difficulties and burdens" (p. 6), estes exibiam uma atitude mais distante em relação ao $\mathrm{PH}$, uma maior resistência e ceticismo quanto à importância de sua presença, e, nalguns casos, encarando sua intervenção como "embaraçosa" ou como "uma infantilidade". A mesma autora -explorando a perspetiva de crianças e adolescentes sobre sua relação com os palhaços ("putting the child's voice in focus") - fala-nos da redução das fronteiras entre o doente e o saudável como resultado da ligação estabelecida entre criança e PH (que a autora designa de "magical attachment"). Conforme Linge, essa relação de vinculação parece funcionar como uma "magical safe area", situada entre a fantasia e a realidade, onde tudo pode acontecer. Segundo a autora, o distanciamento criado em relação aos seus problemas gera um efeito prolongado na vitalidade da criança; incrementando sua autoconfiança, sua esperança e sua capacidade de ver o lado positivo da vida e em relação à sua saúde futura (Linge, 2011, 2012).

Essas mais-valias, apontadas pela literatura, são também reconhecidas pelos profissionais de saúde. Apesar de haver ainda poucos estudos sobre a percepção e opinião desses profissionais, os existentes apontam nesse sentido. Por exemplo, Battrick, Glasper, Prudhoe e Weaver (2007), no seu estudo, procuraram conhecer, entre outras, quais as percepções dos médicos e das enfermeiras quanto ao trabalho dos $\mathrm{PH}$ e sua eficácia nas crianças hospitalizadas. Esses autores verificaram que os médicos consideram, na sua maioria, que a presença dos $\mathrm{PH}$, e as visitas individuais que realizam, são úteis para as crianças. Por sua vez, as enfermeiras têm a mesma percepção, apontando, inclusive, o impacto positivo que estes têm na criança.

Numa tentativa de colmatar um pouco essa falta de dados e conhecer mais profundamente as percepções dos profissionais de pediatria relativamente à futura intervenção dos $\mathrm{PH}$ em seu local de trabalho, as autoras realizaram um estudo cuja metodologia e principais resultados serão, em seguida, apresentados.

\section{Método}

\section{Enquadramento}

Fundada em 2002, a Operação Nariz Vermelho (ONV) representa, em Portugal, a mais antiga associação de palhaços profissionais, e a única a intervir de forma continuada (43 semanas por ano) em contexto pediátrico. A sua intervenção ocorre nos serviços pediátricos de 13 hospitais portugueses.

Um mês antes do início da parceria com os Serviços de Pediatria (SP) de um hospital do Norte de Portugal, realizou-se a avaliação das percepções de seus profissionais quanto às vantagens e desvantagens do trabalho dos $\mathrm{PH}$ junto da criança/adolescente, seus pais/acompanhantes, equipe profissional e junto da própria instituição hospitalar. 


\section{Instrumento}

As percepções dos profissionais de pediatria foram exploradas por intermédio de uma entrevista semiestruturada individual, dirigindo, a cada entrevistado, as seguintes questões: (i) Quais as representações que possui acerca da ONV? (por exemplo, seus contextos de atuação? Formação de seus artistas? Trabalho voluntário ou remunerado?); (ii) Quais as vantagens que antecipa na intervenção dos "Doutores Palhaços" (DP) junto de: (a) usuário pediátrico, (b) e seus pais/acompanhantes, (c) a equipe de saúde; e a própria (d) instituição hospitalar)?; (iii) Quais as maiores dificuldades que espera ocorrer com a presença dos DP junto de: (a) usuário pediátrico (b) seus pais/ acompanhantes (c) equipe de saúde e o próprio (d) hospital?; (iv) Como antevê a articulação de seu trabalho com o dos DP (Será viável? Profícua? Facilitadora de alguns processos)?; (v) O que espera aprender com a presença dos DP em sua unidade de saúde (por intermédio da observação de suas práticas e/ou do trabalho de colaboração desenvolvido entre ambos)?; (vi) Em que medida faz sentido, para si (numa escala de 1 a 10), assumir os DP como mais um elemento da equipa de cuidados dos Serviços de Pediatria desse hospital?; (vii) Que funções/papéis julga que os DP deverão assumir nesses Serviços? (viii) Quais as maiores mudanças que espera encontrar nesse Serviço daqui a dois anos? (Em que áreas/dimensões? Junto de que alvos?)

\section{Participantes}

O estudo envolveu 34 participantes, selecionados com base num método de amostragem por conveniência, segundo a disponibilidade dos profissionais de pediatria para participar nas entrevistas, bem como a sua distribuição representativa em função de sua área profissional e da unidade pediátrica onde exerciam suas funções. $\mathrm{O}$ estudo contou, assim, com médicos $(\mathrm{n}=6)$ enfermeiros $(n=15)$ assistentes operacionais $(n=9)$, administrativos/ secretárias de unidade $(\mathrm{n}=2)$ e vigilantes/seguranças $(\mathrm{n}=2)$. A maioria dos participantes era do gênero feminino $(85,3 \%$ mulheres $-\mathrm{n}=29$; e $14,7 \%$ homens $-\mathrm{n}=5$ ), sendo a média de seus anos de serviço em pediatria de 15 anos. Quanto à unidade pediátrica onde exerciam sua atividade, cinco trabalhavam na Unidade de Neonatologia, quatro no Internamento de Crianças, sete no Internamento de Adolescentes, cinco no Serviço de Urgência Pediátrica, sete no Serviço de Consultas Externas e dois vigilantes trabalhavam em todo o Hospital. Refira-se que quatro enfermeiros trabalhavam em simultâneo no Internamento de Crianças e de Adolescentes.

\section{Procedimentos}

Numa primeira fase, foi pedida autorização para a realização do estudo junto da Comissão de Ética do Hospital. Uma vez aprovado, foram agendadas as entrevistas, junto da chefe dos Serviços Pediátricos, tendo estas ocorrido entre julho e setembro de 2010. As entrevistas foram realizadas em um local que reunia condições para assegurar a privacidade dos participantes e a não interrupção do processo de coleta de dados. Junto de cada participante foram clarificados os objetivos e o caráter voluntário e confidencial do estudo, seguidos da obtenção de seu consentimento informado. As entrevistas, em média, duraram 25 minutos. Estas foram registadas em áudio e posteriormente transcritas verbatim.

\section{Tratamento dos dados}

Após a transcrição das entrevistas, foi feita a análise de conteúdos, pelos cinco investigadores envolvidos em sua coleta. Posteriormente, procedeu-se ao acordo entre avaliadores, com recurso a dois elementos independentes. Foi também feita uma abordagem quantitativa aos resultados, através do cálculo da frequência das respostas dos participantes nas diferentes categorias emergidas.

\section{Resultados}

Nas tabelas 1 e 2, apresentam-se, respetivamente, as expetativas dos participantes em relação às vantagens e desvantagens da intervenção dos $\mathrm{PH}$ junto da criança e adolescente. Nestes consta informação acerca de: (i) a frequência com que cada uma das categorias/ subcategorias foi referida, e sua percentagem relativa (em função do número total de participantes); (ii) a média do número de vantagens e desvantagens referidas; e (iii) o número mínimo e máximo de vantagens e desvantagens. Para fins de ilustração do discurso dos participantes, são dados alguns exemplos de resposta.

Vantagens da presença dos PH nos SP junto das crianças/adolescentes

Dentre as vantagens apontadas à presença dos $\mathrm{PH}$ junto das crianças e adolescentes, salientam-se as referentes à recreação e entretenimento $(n=27)$. Menciona-se, também, o impacto positivo esperado ao nível da redução do tédio experimentando nos momentos de 
Tabela 1. Vantagens/mais-valias da intervenção dos PH junto das crianças/ adolescentes

\begin{tabular}{llcc}
\hline Categorização das respostas & $\mathrm{N}$ & $\%$ \\
\hline Amenização da internação & & 12 & $35,3 \%$ \\
\hline Amenização do tratamento & $\begin{array}{l}\text { Abstração/atenuar da dor aquando da aplicação de } \\
\text { tratamentos invasivos } \\
\text { Desmistificação do tratamento e/ou dos } \\
\text { profissionais de saúde }\end{array}$ & 5 & $14,7 \%$ \\
& Facilitação do processo/Maior colaboração nos & $26,5 \%$ \\
& tratamentos & 6 & $17,6 \%$ \\
\hline Amenização do impacto emocional & Ventilação emocional; catarse & 3 & $8,8 \%$ \\
negativo & Diminuição da tristeza/depressão & 7 & $20,6 \%$ \\
& Redução do medo e da ansiedade & 5 & $14,7 \%$ \\
\hline Recreação/Lúdico & O alegrar da criança & 14 & $41,2 \%$ \\
& Redução do tempo de espera/ “enfado” & 13 & $38,2 \%$ \\
\hline
\end{tabular}

espera e nos muitos momentos inocupados que marcam a experiência de internação $(\mathrm{n}=13)$. De acrescentar que cada profissional apontou, em média, 2.29 vantagens, variando as respostas do grupo de participantes entre uma e cinco vantagens. Quanto aos contributos esperados à "amenização do tratamento", 20 profissionais referiram vantagens ao nível da desmistificação do tratamento e/ou dos profissionais de saúde; da diminuição do medo e rejeição em relação às "batas ${ }^{1}$ brancas" e dos cuidados por estes prestados ( $\mathrm{n}=9)$. Eis um exemplo recolhido nas respostas dos participantes: "A maior parte deles [crianças] não nos aceitam, porque estão com medo das picas ${ }^{2}$, da... olham para tudo, ficam com medo, não é?...e que eles tenham algum contacto antes ... com algumas brincadeiras, talvez nos ajude [...]”.

Ainda nesta categoria, foi salientado o provável contributo dos $\mathrm{PH}$ para a maior colaboração da criança/adolescente nos tratamentos $(\mathrm{n}=6)$, tal como ilustra o seguinte exemplo: "[...] por um lado podem ser muito positivos e temos a experiência de que se fizermos algumas "palhaşadas", com bonecos e falarmos com eles quando estão sendo intervencionados ${ }^{3}$, eles toleram muito melhor, são mais cooperantes...". Com menor frequência $(n=5)$ surgiram, nesta categoria, as referências feitas ao contributo dos $\mathrm{PH}$ à diminuição das dores associadas à condição clínica da criança/adolescente ou aquando da aplicação de

\footnotetext{
${ }^{1}$ No português do Brasil, "jalecos"

${ }^{2}$ No português do Brasil: "picadas"

${ }^{3}$ Entende-se por "intervencionados", os procedimentos médicos

Psico-USF, Bragança Paulista, v. 19, n. 3, p. 377-386, set./ dez. 2014
}

tratamentos invasivos. Tal redução deve-se a uma maior abstração, pela criança, em relação aos tratamentos a que está sendo sujeita, porque centrada na interação com os palhaços.

Alguns participantes referiram, também, o caráter mais positivo, menos traumático que a presença dos $\mathrm{PH}$ poderia conferir à experiência de internação da criança/ adolescente $(\mathrm{n}=12)$, amenizando, inclusive, sua representação do hospital como um ambiente hostil. Foi também sinalizada a "amenização do impacto emocional negativo", que a presença dos $\mathrm{PH}$ poderia ter sobre a criança/adolescente $(n=15)$. Segundo as respostas de sete profissionais (20\% do grupo total), a presença dos $\mathrm{PH}$ poderá contribuir para (i) a diminuição da tristeza (e, em alguns casos, depressão) provocada pela sua condição médica, internação ou outras experiências de adversidade vivenciadas nesse processo; (ii) a diminuição dos medos e ansiedade experienciados; e (iii) a redução da tensão. A presença dos $\mathrm{PH}$ foi igualmente apontada como promotora de processos de catarse/ventilação emocional $(\mathrm{n}=3)$, da qual é exemplo a seguinte resposta:"[...] a criança pode estar deprimida e, então, se entrar um palhaço, a criança pode-se libertar de muita coisa de que naquele momento não está a conseguir".

\section{Dificuldades junto das crianças e adolescentes}

$\mathrm{Na}$ Tabela 2, apresentam-se as dificuldades apontadas à presença dos $\mathrm{PH}$ junto das crianças $\mathrm{e}$ adolescentes. A incidência das respostas por categoria/subcategoria são representadas em termos de sua 
frequência. Observe-se que o número de desvantagens variou entre 0 e 3 por participante, registando-se uma média por sujeito inferior à unidade $(\mathrm{M}=0.73)$.

De entre as categorias, surgiram: o (i) "Medo/ Pânico do palhaço" por parte da criança; (ii) a "Pouca receptividade", por estar em sofrimento; (iii) a resistência à presença dos $\mathrm{PH}$ pelo adolescente; e, finalmente, (iv) a alusão a alguns fatores de ordem externa ao usuário pediátrico. Observe-se que 41,2\% desses profissionais explicitaram não antecipar quaisquer dificuldades associadas à presença dos $\mathrm{PH}$ neste contexto, quer junto das crianças, quer dos adolescentes.

Como se pode verificar pela leitura da Tabela 2, metade dos profissionais entrevistados $(n=12)$ aponta desvantagens para a criança, salientando-se o medo ou pânico em relação à figura do palhaço $(35,3 \%)$. Outros $14,7 \%(n=5)$ apontam a resistência da criança à presença dos $\mathrm{PH}$ pelo fato de se encontrar em sofrimento e, por isso, menos receptiva.

Quanto aos adolescentes, as desvantagens prenderam-se com o fato de estes poderem encarar a figura e as brincadeiras dos PH com uma "infantilidade" $(n=3)$.

Por último, observou-se a alusão, por quatro participantes, ao fato de a presença dos $\mathrm{PH}$ não ser possível, em situações de doença infetocontagiosa.

\section{Discussão}

Após a apresentação dos resultados, passa-se à sua discussão e apreciação crítica, tendo como referência as evidências apontadas por outros trabalhos na área e aqueles que foram os dados recolhidos no âmbito da presente pesquisa.

Quanto às vantagens da intervenção dos $\mathrm{PH}$ junto das crianças/adolescentes, os profissionais entrevistados salientaram sua dimensão lúdica, servindo a objetivos como a distração e o entretenimento (com especial ênfase na criança). Destacaram-se, também, os contributos dos $\mathrm{PH}$ para o amenizar da internação e dos tratamentos recebidos, bem como para o minorar do impacto emocional negativo que a condição clínica, tratamentos e internação poderão ter sobre a criança/ adolescente. A redução da percepção de demora ou tédio quando da hospitalização ou em situações de espera foi também referido por $40 \%$ desses profissionais, que anteciparam que a presença dos $\mathrm{PH}$ poderá aumentar a tolerância e cooperação do usuário pediátrico e seus pais/acompanhantes nesses momentos. Perto de $1 / 3$ dos profissionais antecipou, também, a promoção, pelos $\mathrm{PH}$, de experiências mais positivas de hospitalização, tornando-a menos desagradável e/ou "traumática", e dando lugar a representações, pela criança, do hospital como um ambiente menos hostil. Tais dados vão ao encontro das evidências recolhidas por Wuo (1999), que relata os contributos dos $\mathrm{PH}$ para o amenizar dos traumas causados pela internação. Segundo essa autora, a presença dos $\mathrm{PH}$ pode ser "transformadora", convertendo alguns elementos negativos da doença e da hospitalização em elementos (ou momentos) positivos, representando a transição da angústia para a alegria. Assim, tal como referem Valladares e Carvalho (2006), através de uma abordagem lúdica, os palhaços parecem contribuir para a neutralização de alguns dos fatores emocionais negativos associados à doença e à hospitalização, exercendo um efeito preventivo, uma vez que ajudam a evitar o emergir e/ou instalação de algumas disfunções, nomeadamente os "traumas" de que nos falam Parcianello e Felin (2008). As evidências e leituras propostas pelo trabalho desses autores parecem permitir interpretações consonantes com as percepções dos profissionais entrevistados nesta pesquisa.

Outra vantagem antecipada com a presença dos PH (referida por 58,8\% dos participantes) prendeu-se com a "amenização dos tratamentos", designadamente ao nível da sua desmistificação e diminuição dos medos e comportamentos de rejeição e de baixa cooperação

Tabela 2. Dificuldades associadas à presença dos ph junto das crianças/ adolescentes

\begin{tabular}{llcc}
\hline Categorização das respostas & & $\mathrm{n}$ & $\%$ \\
\hline Nenhuma & Não antecipam dificuldades & 14 & 41,2 \\
\hline Crianças & Medo/pânico do Palhaço & 12 & 35,3 \\
& Pouca receptividade por estarem em sofrimento & 5 & 14,7 \\
\hline Adolescentes & Pouca receptividade/infantilidade & 3 & 8,8 \\
\hline Dificuldades extrínsecas & Pacientes com doenças infetocontagiosas & 4 & 11,8 \\
\hline
\end{tabular}


com a equipe de saúde. Assim, à semelhança dos relatos de Masetti (2003) e Melo (2007), no âmbito dos seus trabalhos de pesquisa, a intervenção dos $\mathrm{PH}$ foi antecipada pelos participantes deste estudo como potencial promotora da colaboração da criança com os exames e tratamentos, da maior aceitação de determinados procedimentos, e/ou da melhoria da comunicação e da qualidade das interações estabelecidas entre a criança e seus cuidadores.

Outro aspeto que se destacou no discurso dos profissionais entrevistados foi o possível contributo dos PH para a diminuição das dores associadas à condição clínica do paciente ou à aplicação de tratamentos invasivos, justificado por uma maior abstração da criança/adolescente em relação aos tratamentos, uma vez que está focada nos PH. Evidências semelhantes foram salientadas pelos estudos de Lima e cols. (2007), de Martins e Paduan (2010) e de Araújo e Guimarães (2009) - esse último com crianças e adolescentes com câncer. Também esses mostraram estudos que o contacto com os $\mathrm{PH}$ se revelou negativamente correlacionado com os níveis de dor experienciados, bem como, nos casos oncológicos, com as náuseas associadas aos tratamentos quimioterapêuticos.

Os contributos dos PH para a diminuição de afetos negativos como a tristeza, o medo, ansiedade ou depressão aparecem, também, no discurso desses profissionais. De fato, tal como referem Mazur e cols. (2005, p.14): "Além da angústia de ficar longe de sua família, a criança pode expressar o medo do desconhecido e da solidão, como a desconfiança de quem a cuida, entre outros sentimentos." Assim, apar da alegria, os participantes anteciparam que a presença dos $\mathrm{PH}$ pode ser promotora de processos de catarse/ventilação emocional, sendo que o riso e o humor poderão ter um efeito libertador de algumas tensões associadas à situação clínica da criança/adolescente, à sua internação e/ou a outras experiências de adversidade vividas em contexto hospitalar. Em conformidade com as ideias de alguns profissionais desse estudo, Wuo (1999) defende que $\mathrm{o}$ intenso prazer experienciado pelas crianças em suas brincadeiras com os $\mathrm{PH}$ decorre não apenas do prazer de brincar, mas, também, do fato de, ao brincar, encontrarem um meio de catarse e domínio de suas angústias. Masetti (2011) assume, também, o riso e o humor como aspetos determinantes na recuperação física e equilíbrio emocional do paciente pediátrico, e como um recurso importante no enfrentamento da doença, uma vez que permitindo o acesso a fatos que, tal como afirma “... por obstáculos pessoais, não poderiam se revelar de forma aberta e consciente" (p. 51). O acesso a esses fatos permite, segundo a autora, a posterior recondução da energia despendida para lidar emocionalmente com a doença para outros aspetos importantes à sua recuperação física. Assim, a presença dos $\mathrm{PH}$, ao permitir que a criança se "liberte" das emoções negativas associadas ao processo em curso, parece torná-la mais "disponível" para a recuperação.

Ao olhar destes autores e dos profissionais que participaram neste estudo, é importante acrescentaras evidências recolhidas por outros pesquisadores da área, os quais apontam para as melhorias resultantes da presença dos $\mathrm{PH}$ ao nível da disposição e atitude do paciente pediátrico (mais calmo, mais feliz, com maior autoestima, mais otimista...); o aumento da expressão de emoções, como o riso, alegria e humor, ou o incremento da percepção de controle (Fernandes \& Arriaga, 2010; Kingsnorth, Blain, \& McKeever, 2010; Masetti, 1998; Melo, 2007), todos eles importantes para a recuperação do paciente pediátrico e para a diminuição das "sequelas emocionais" que os processos de doença e internação poderão deixar ficar.

Numa análise global das vantagens apontadas pela presença dos $\mathrm{PH}$ em contexto pediátrico, verifica-se que é (quantitativa e qualitativamente) amplo o leque de percepções de benefícios que esses profissionais têm relativamente aos potenciais contributos do $\mathrm{PH}$ para o bem-estar da criança/adolescente. De entre estas, o amenizar das experiências (físicas e emocionais) vividas no hospital e o "importar" de uma componente lúdica e recreativa para este cenário, (rumo a uma maior humanização dos cuidados pediátricos), merecem particular destaque, indo estas ao encontro de alguns dos objetivos estruturantes da ONV ao nível da intervenção de seus artistas.

Olhando as dificuldades antecipadas pelos profissionais de saúde entrevistados como consequência da presença dos $\mathrm{PH}$ nos serviços de pediatria, observou-se um cenário distinto do anterior, nomeadamente ao nível da quantidade e diversidade de seu discurso. Assim, foi menor o leque de dificuldades antecipadas $\mathrm{e}$, inclusive, alguns deles não anteviram qualquer tipo de dificuldade decorrente dessa colaboração. O presente cenário espelha, tal como já referido, a grande abertura habitualmente existente entre a comunidade hospitalar relativamente à presença dos $\mathrm{PH}$. Existem, no entanto, alguns obstáculos e/ou resistências, mesmo que pontuais, à semelhança do observado noutros estudos (Fernandes \& Arriaga, 2010; Vagnoli e cols. 2005). No presente trabalho, as dificuldades associaram-se às 
respostas de medo ou pânico por parte da criança e/ ou resistência à presença do $\mathrm{PH}$, pelo fato de se encontrarem em sofrimento. Quanto aos adolescentes, as desvantagens prenderam-se com a franca probabilidade de estes encararem a figura e as brincadeiras dos $\mathrm{PH}$ como uma "infantilidade", tendo o mesmo tipo de evidências sido identificadas por Linge (2012).

\section{Considerações finais}

As reflexões dos participantes em torno das potenciais mais-valias da futura presença dos $\mathrm{PH}$ nos serviços de pediatria de seu hospital deram a conhecer um amplo leque de expetativas, maioritariamente positivas. Dentre elas, destacam-se os contributos esperados à amenização da experiência de internação pela criança/adolescente, quer no sentido de minorar algumas das sequelas emocionais que o internamento, a doença e todo o sofrimento e vulnerabilidade associados por vezes deixam ficar, quer no sentido da construção de representações mais positivas da doença, hospital, tratamentos e/ou dos seus cuidadores hospitalares. A humanização das relações dos serviços e dos cuidados surgem, também, entre as expetativas desses profissionais quando antecipando os $\mathrm{PH}$ como mais um dos atores do universo hospitalar. A sua presença é entendida, por alguns, como, por si só, humanizadora. Efetivamente, se por um lado, através da brincadeira e do humor, o PH traz o riso, a alegria, quebra "gelos" ou estabelece "pontes" entre os seus diferentes atores; por outro, contém algumas oportunidades de aprendizagem. Tais oportunidades encontram-se nos processos de colaboração e/ou de observação de outros saberes, ferramentas ou estratégias (do "fazer", do "ser" e do "estar" nos cuidados) que os $\mathrm{PH}$ trazem para o cenário hospitalar. Para além de facilitadoras de suas práticas, tais aprendizagens são encaradas, por alguns dos profissionais entrevistados, como potenciadoras do bem-estar e dos processos de recuperação do paciente pediátrico, bem como da qualidade e sanidade dos contextos e dos agentes responsáveis por seus cuidados (nomeadamente os profissionais de saúde e seus pais). Assim sendo, menor dor, ansiedade, tristeza, resistência aos tratamentos e aos profissionais; melhor e mais rápida recuperação do paciente pediátrico; ou, uma maior satisfação e melhor "qualidade de vida" em contexto hospitalar são antecipados como algumas das vantagens resultantes da presença dos $\mathrm{PH}$ na ala pediátrica.

Para terminar, destaque-se que, muito embora os dados divulgados neste artigo se centrem num contexto específico, eles dão a conhecer um olhar francamente positivo - por parte dos profissionais de pediatria - quanto ao contributo dos PH para a qualidade da vivência da hospitalização infantil. Tal parece abrir algum espaço para o estabelecimento de práticas colaborativas entre os profissionais de saúde e os $\mathrm{PH}$, e para a implementação de intervenções conjuntas que permitam capacitar o paciente pediátrico - e todos os agentes responsáveis por seus cuidados (pais, profissionais de saúde, etc.) - para enfrentar as dificuldades físicas e psicológicas experienciadas; satisfazer suas necessidades psicológicas, afetivas e sociais durante a hospitalização; e reduzir o impacto negativo que essas experiências poderão ter em seu desenvolvimento e bem-estar (atual e futuro).

\section{Referências}

Araújo, T. C., \& Guimarães, T. B. (2009). Interações entre voluntários e usuários em onco-hematologia pediátrica: um estudo sobre os "palhaços-doutores”. Estudos e Pesquisas em Psicologia, 9(3), 632-647.

Azevedo, D., Santos, J., Justino, M. A., Miranda, F., \& Simpson, C. (2007). O brincar como instrumento terapêutico na visão da equipe de saúde. Ciência, Cuidado e Saúde, 6(3), 335-341.

Barros, L. (1998). As consequências psicológicas da hospitalização infantil: Prevenção e controle. Análise Psicológica, 15(1), 11-28.

Battrick, C., Glasper, E. A., Prudhoe, G., \& Weaver, K. (2007). Clown humour: the perceptions of doctors, nurses, parents and children. Journal of Children's and Young People's Nursing, 1(4), 174-179.

Bess d'Alcantara, E. (2008). Criança hospitalizada: o impacto do ambiente hospitalar no seu equilíbrio emocional. Revista Virtual de Psicologia Hospitalar e Saúde, 3(6), 38-55.

Brewer, S., Gleditsch, S. L., Syblik, D., Tietjens, M. E., \& Vacik, H. W. (2006). Pediatric Anxiety: child life intervention in day surgery. Journal of Pediatric Nursing, 21(1), 13-22.

Broering, C. V., \& Crepaldi, M. A. (2011). Preparação psicológica e o estresse de crianças submetidas a cirurgias. Psicologia em Estudo, 16(1), 15-23.

Caires, S., Dias, M. F., Esteves, C. H., Belo, S., Correia, S., Diaz, Z., \& Ballester, S. (2010). Rir é o melhor remédio? O bumor no contexto hospitalar. Comunicação 
apresentada no Congresso Emoções na Saúde, Ourém, 22 e 23 out. 2010.

Duffin, C. (2009). Send in the clowns. Nursing Management, 16(3), 22-24.

Fernandes, S. (2008). Os efeitos da presença de palhaços nos estados emocionais e na redução das preocupações em crianças submetidas a intervenção cirúrgica. Comunicação apresentada no Seminário de Investigação em Psicologia, Aconselhamento e Psicoterapia, Universidade Lusófona de Humanidades e Tecnologias.

Fernandes, S., \& Arriaga, P. (2010). The effects of clown intervention on worries and emocional responses in children undergoing surgery. Jornal Health Psycho$\log y, 15,405-415$.

Garcia, D. T. R., Silva, J. G., Vaz, A. C., Filocomo, F. R. F., \& Filipini, S. M. (2009). A influência da terapia do riso no tratamento do paciente pediátrico. Disponível em: http://www.inicepg.univap.br/cd/ INIC_2009/anais/arquivos/0918_0498_01.pdf. Acesso em 9 de jan. 2011.

Gryski, C. (2003). Stepping over thresholds. A personal meditation on the work and play of the therapeutic clown. POIESIS: A Journal of the Arts and Communication, 5, 94-99.

Hart, R., \& Walton, M. (2010). Magic as a Therapeutic intervention to promote coping in hospitalized pediatric patients. Pediatric Nursing, 36(1), 11-17.

Kingsnorth, S., Blain, S., \& McKeever, P. (2010). Physiological and emotional responses of disabled children to therapeutic clowns: a pilot study. Evidence-Based Complementary and Alternative Medicine (2011). Disponível: http://downloads.hindawi. com/journals/ecam/2011/732394.pdf. Acesso em: 23 mar. 2012.

Kumamoto, L. H. M. C. C., Barros, R. C. N., Carvalho, T. C. T. D., Gedelha, E. C. M., \& Costa, R. R. L. O. (2004). Apoio à criança hospitalizada: proposta de intervenção lúdica. Comunicação apresentada no $2^{\circ}$ Congresso Brasileiro de Extensão Universitária, Belo Horizonte. Disponível em: http://www. ufmg.br/congrext/Saude/Saude23.pdf. Acesso em: 23 de mar. 2012.

Lima, R., Azevedo, E., Nascimento, L., \& Rocha, S. (2009). A arte do teatro clown no cuidado às crianças hospitalizadas. Revista da Escola de Enfermagem-USP, 43(1), 186-193.
Linge, L. (2011). Joy without demands: hospital clowns in the world of ailing children. Int. J.Qua.l Stud. Health Well-being, 6(1).

Linge, L. (2012). Magical attachment: children in magical relations with hospital clowns. Int. J. Qual. Stud. Health Well-being, 7.

Martins, S. T. F., \& Paduan, V. C. (2010). A equipe de saúde como mediadora no desenvolvimento psicossocial da criança hospitalizada. Psicologia em Estudo, 15(1), 45-54.

Masetti, M. (1998). Solucões de palhaços: transformacõos na realidade hospitalar. São Paulo: Palas Athena.

Masetti, M. (2003). Boas misturas: MMD Editores.

Masetti, M. (2011). Ética da alegria. Sâo Paulo: Sinergias.

Mazur, A., Batista, G. L., Andreatta, D., Ribas, M., \& Campos, T. (2005). O Processo de hospitalização da criança sob a ótica do familiar. (v.25). http:// bvsms.saude.gov.br/bvs/publicacoes/57cbe/resumos/834.htm. Acesso em: 25 mar. 2011.

Meisel, V., Chellew, K., Ponsell, E., Ferreira, A., Bordas, L., \& García-Banda, G. (2009). The effect of "hospital clowns" on distress and maladaptive behaviours of children who are undergoing minor surgery. Psicothema, 21(4), 604-609.

Melo, A. (2007). A terapêutica artística promovendo saúde na instituição hospitalar. Ibérica: Revista Interdisciplinar de Estudos Ibéricos e Ibero-Americanos, 3, 159-189.

Mitre, R., \& Gomes, R. (2004). A promoção do brincar no contexto da hospitalização infantil como ação de saúde. Ciência \& Saúde Coletiva, 9(1), 147-154.

Motta, A., \& Enumo, S. R. F. (2004). Brincar no hospital: estratégia de enfrentamento da hospitalização infantil. Psicologia em Estudo, 9(1), 19-28.

Mussa, C., \& Malerbi, F. E. K. (2008). O impacto da actividade lúdica sobre o bem-estar de crianças hospitalizadas. Psicologia: Teoria e Prática, 10(2), 83-93.

Oliveira, L. D. B., Gabarra, L. M., Marcon, C., Silva, J. L. C., \& Macchiaverni, J. (2009). A brinquedoteca hospitalar como factor de promoção no desenvolvimento infantil: relato de experiência. Rev. Bras. Crescimento Desenvolvimento Hum., 19(2), 306-312. 
Parcianello, A. T., \& Felin, R. B. (2008). E agora doutor, onde vou brincar? Considerações sobre a hospitalização infantil. Barbarói, Santa Cruz do Sul, 28, 147-166.

Redondeiro, M. (2003). O quotidiano hospitalar da criança: constrangimentos e possibilidades de desenvolvimento. (Dissertação de Mestrado), Universidade do Minho, Braga.

Schmitz, S. M., Piccoli, M., \& Vieria, C. S. (2003). A criança hospitalizada, a cirurgia e o brinquedo terapêutico: uma reflexão para a enfermagem. Ciência, Cuidado e Saúde, 2(1), 67-73.

Tavares, P. (2008). Acolher brincando, a brincadeira terapêutica no acolbimento de enfermagem à criança hospitalizada. (Dissertação de Mestrado em Ciências de Enfermagem), Universidade do Porto, Porto.
Valladares, A., \& Carvalho, A. (2006). A arterapia e o desenvolvimento do comportamento no contexto da hospitalização. Revista da Escola de Enfermagem USP, 40(3), 350-355.

Vieira, M. A., \& Lima, R. A. G. (2002). Crianças e adolescentes com doença crônica: convivendo com mudanças. Revista Latino-Americana de Enfermagem, 10(4), 552-560.

Wuo, A. E. (1999). O Clown Visitador no tratamento de crianças hospitalizadas. Univeridade Estadual de Campinas, Campinas.

Recebido em: 03/01/2014

Reformulado em: 13/05/2014

Aprovado em: 30/06/2014

Sobre as autoras:

Susana Caires é doutora em Psicologia da Educação pela Universidade do Minho e professora auxiliar do Instituto de Educação dessa Universidade. Coordena o projeto de investigação "Rir é o Melhor Remédio?”, que explora o impacto da intervenção dos palhaços de hospital em pediatria.

Carla Hiolanda Esteves é mestre em Educação pela Universidade do Minho - Portugal, e doutoranda em Psicologia da Educação, nessa universidade. Integra o projeto de investigação "Rir é o melhor remédio?", explorando o impacto da intervenção dos palhaços de hospital nas crianças hospitalizadas.

Susana Correia é mestre em Psicologia da Educação, pela Universidade de Évora - Portugal. A sua dissertação de Mestrado tem como tema: "Vivências do Pai com Filho(a) em Situação de Internamento Hospitalar". Integra o projeto de investigação "Rir é o Melhor Remédio?".

Isabel Almeida é mestre em Intervenção Psicossocial com Crianças, Jovens e Famílias, pela Universidade do Minho. Trabalha numa Instituição de Acolhimento de Crianças e Jovens em risco. Recentemente tem direcionado o seu interesse para o impacto do humor nas vivências emocionais das crianças.

Contato com as autoras:

Instituto de Educação. Universidade do Minho - Braga

Prof $f^{a}$. Susana Caires

Instituto de Educação da Universidade do Minho

Campus de Gualtar

4710-057 Braga

Portugal 